\title{
On an Asymptotic Expansion of the Hypergeometric Function.
}

\author{
By Dr T. M. MacRobert.
}

(Read and Received 7th December 1928.)

In a previous paper* the author has employed the expansion

where

$$
F(\alpha, \beta, \gamma, z)=\sum_{r=1}^{s} T_{r}+P_{s}
$$

$$
\begin{gathered}
T_{r}=\frac{\alpha(\alpha+1) \ldots(\dot{\alpha}+r-2) \beta(\beta+1) \ldots(\beta+r-2)}{(r-1) ! \gamma(\gamma+1) \ldots(\gamma+r-2)} z^{r-1}, \\
P_{s}=\frac{1}{B(\beta+s, \gamma-\beta)} T_{s+1} \int_{0}^{1} s(1-t)^{s-1} I d t,
\end{gathered}
$$

and $\dagger \quad I=\int_{0}^{1} \xi^{\beta+s-1}(1-\zeta)^{\gamma-\beta-1}\left(1-\zeta(z)^{-\alpha-8} d \xi\right.$,

to establish the theorem that, if $-\pi / 2<a m p \gamma<\pi / 2$, the function $P_{s} / T_{s+1}$ remains finite as $\gamma \rightarrow \infty$. This theorem is valid provided that $z$ is not real and $\geqq 1$.

It will now be shown that the theorem is true for a more extended range of values of $a m p \gamma$.

In the integral $I$ put $\zeta=1-e^{-\lambda \mp}$; then

$$
I=\int_{0}^{\infty}\left(1-e^{-\lambda}\right)^{\beta+s-1} e^{-\lambda(\gamma-\beta)}\left\{1-t z\left(1-e^{-\lambda}\right)\right\}^{-\alpha-s} d \lambda
$$

the path of integration being the real axis from 0 to $\infty$. This path may now be deformed into that straight line from the origin to infinity which makes an acute angle $-\phi$ with the positive real axis, and the integral is still convergent provided that, on the path of integration, $1-t z\left(1-e^{-\lambda}\right) \neq 0$, and provided that

$$
-\pi / 2<a m p(\lambda \gamma)<\pi / 2 \text {. }
$$

* Proc. Edin. Math. Soc., Vol. XLI., pp. 82-92.

$+K(\gamma)$ and $s$ are taken so large that $R(\gamma-\beta)>0$ and $R(\beta+\gamma)>0$.

$\mp$ Cf. G. N. Watson, Trans. Camb. Phil. Soc., Vol. 22, 1918, p. 299. 
Since $a m p \lambda=-\phi$ the latter inequality may be written

$$
-\pi / 2+\phi<\operatorname{amp} \gamma<\pi / 2+\phi
$$

and, by reversing the transformation $\zeta=1-e^{-\lambda}$, it can be made clear that the first condition may be replaced by the proviso that $1-t z \zeta$ must not vanish at any point on the contour in the $\zeta$-plane which corresponds to the path of integration in the $\lambda$-plane.

To determine this contour put $\lambda=\mu(1-i t)$, where $\mu$ is real and $t=\tan \phi ;$ then

$$
\zeta=\xi+i \eta=1-e^{-\lambda}=1-e^{-\mu+i \mu t}
$$

so that

$$
\xi=1-e^{-\mu} \cos \mu t, \eta=-e^{-\mu} \sin \mu t .
$$

Hence, if $(r, \theta)$ are the polar coordinates of the point $(\xi, \eta)$ referred to axes parallel to the $\xi$ and $\eta$ axes and passing through the point $(1,0)$ in the $\zeta$-plane

$$
r=e^{-\mu}, \tan \theta=\tan \mu t,
$$

and the contour is an equiangular spiral (see Fig.) whose equation may be written

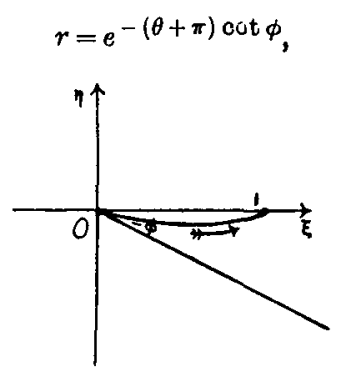

where $\theta=-\pi$ and $r=1$ when $\mu=0, \lambda=0, \zeta=0$; at this point $\frac{d \xi}{d \lambda}=1$, so that the contour makes an angle $-\phi$ with the $\xi$ axis, and the path is described from $\zeta=0$ in the direction indicated by the arrow.

It can easily be shown that the entire contour of integration lies between the lines $a m p \xi= \pm \phi$. Now $1-t z \xi$ must not vanish for any point $\zeta$ on this contour. But, since $0 \leqq t \leqq 1$, the values of $z$ which satisfy $z=1 /(t \zeta)$ will lie in the region to the right of the $\eta$ axis which is bounded by the lines $a m p \zeta= \pm \phi$. Also the 
hypergeometric expansion is valid within the unit circle; hence the expansion ( 1 ) is valid for

$$
-\pi / 2+\phi<\operatorname{amp} \gamma<\pi / 2+\phi
$$

at all points external to a region $B$ which is bounded by the lines $a m p \zeta= \pm \phi$ and the circle $|\zeta|=1$.

But if $z$ is any interior point of the region $A$ consisting of the entire complex plane bounded by a cross-cut along the positive real axis from +1 to $+\infty, \phi$ can be chosen so small $(<|\operatorname{amp} z|)$ that $z$ does not lie in $B$. Hence for any interior point of $A$ a $\phi$ can be found such that the expansion defined by (1) and (2) is valid for $-\pi / 2+\phi<a m p \gamma<\pi / 2+\phi$.

It remains to prove that the theorem is true under these conditions. Now, for points on the path of integration in the $\lambda$-plane

$$
\begin{aligned}
\left|1-e^{\lambda}\right| & =\sqrt{ }\left(1-2 e^{-\mu} \cos \mu t+e^{-2 \mu}\right) \\
& =\sqrt{ }\left\{\left(1-e^{-\mu}\right)^{2}+2 e^{-\mu}(1-\cos \mu t)\right\} \\
& =\left(1-e^{-\mu}\right) \sqrt{ }\left\{1+\left(\frac{\sin \frac{1}{2} \mu t}{\sinh \frac{1}{2} \mu}\right)^{2}\right\} \\
& <C\left(1-e^{-\mu}\right)
\end{aligned}
$$

where $C$ is a definite positive constant $(|\phi|<\pi / 2)$. Also; let $\gamma=g e^{i \phi}$, so that $-\pi / 2<a m p g<\pi / 2$, and note that $\lambda=\mu \sec \phi e^{-i \phi}$. Then, if $\beta-\sigma+i \tau$ and $g=l+i m$,

$$
R\{\lambda(\gamma-\beta)\}=\mu(l \sec \phi-\sigma-\tau \tan \phi) .
$$

Accordingly, for any point $z$ within the region $A$,

$$
.|I|<K \int_{0}^{\infty}\left(1-e^{-\mu) \sigma+\Delta-1} e^{-\mu(l \sec \phi-\sigma-\tau \tan \phi)} d \mu,\right.
$$

where $K$ is a definite constant. Here put $\xi=1-e^{-\mu}$ and get

thus

$$
\begin{gathered}
|I|<K \int_{0}^{1} \xi^{\sigma+s-1}(1-\xi)^{l \sec \phi-\sigma-\tau \tan \phi-1} d \xi \\
=K B(\sigma+s, l \sec \phi-\sigma-\tau \tan \phi): \\
\left|\frac{P,}{T_{t+1}}\right|<K \frac{B(\sigma+s, l \sec \phi-\sigma-\tau \tan \phi)}{|B(\beta+s, \gamma-\beta)|} \\
=K \frac{\Gamma(\sigma+8)}{|\Gamma(\beta+8)|} \frac{\Gamma(l \sec \phi-\sigma-\tau \tan \phi)}{\Gamma(l \sec \phi-\tau \tan \phi+8)}\left|\frac{\Gamma(\gamma+s)}{\Gamma(\gamma-\beta)}\right|,
\end{gathered}
$$

and when $\gamma \rightarrow \infty, l \rightarrow \infty$, and 
$\left|\frac{P}{T_{s+1}}\right|<K \frac{\Gamma(\sigma+s)}{|\Gamma(\beta+s)|} \frac{|\gamma \beta+s|}{(l \sec \phi)^{\sigma+s}}=K \frac{\Gamma(\sigma+s)}{|\Gamma(\beta+s)|}\left(\frac{|\gamma|}{l \sec \phi}\right)^{\sigma+s} e^{-\chi \tau}$, where $a m p \gamma=\chi$. But $|\gamma| / l$ is finite; hence the theorem holds.

Similarly the theorem can be shown to hold for the region $-\pi / 2-\phi<a m p \gamma<\pi / 2-\phi$; thus it holds for the entire region

$$
-\pi / 2-\phi<\operatorname{amp} \gamma<\pi / 2+\phi .
$$

The Asymptotic Expansion of $P_{n}^{m}(z)$. It follows that, for any interior point $z$ of the region in which the asymptotic expansion of $P_{n}^{m}(z)$ for $n$ large is valid, a $\phi$ can be found such that the asymptotic expansion holds for $-\pi / 2-\phi<\operatorname{amp} n<\pi / 2+\phi$. Hence, as $P_{-n-1}^{m}(z)=P_{n}^{m}(z)$, the function possesses an asymptotic expansion for any value of $a m p n$.

By means of the formula

$$
Q_{n}^{m}(z)=Q_{-n-1}^{m}(z)+\frac{\pi e^{m \pi i} \cos n \pi}{\sin (n-m) \pi} \frac{\Pi(n+m)}{\Pi(n-m)} P_{-n-1}^{-m}(z)
$$

a similar result can be obtained for the function $Q_{n}^{m}(z)$. 